\title{
Design of nanoscale capacitors based on metallic borophene and insulating boron nitride layers
}

\author{
Y. Mogulkoc $\odot,{ }^{1}$ A. Mogulkoc $\odot,{ }^{2}$ H. E. Guler, ${ }^{2}$ and E. Durgun $\oplus^{3, *}$ \\ ${ }^{1}$ Department of Physics Engineering, Faculty of Engineering, Ankara University, 06100 Tandogan, Ankara, Turkey \\ ${ }^{2}$ Department of Physics, Faculty of Sciences, Ankara University, 06100 Tandogan, Ankara, Turkey \\ ${ }^{3}$ UNAM-National Nanotechnology Research Center, Institute of Materials Science and Nanotechnology, Bilkent University, \\ Ankara 06800, Turkey
}

(Received 28 February 2021; revised 2 October 2021; accepted 24 November 2021; published 13 December 2021)

\begin{abstract}
In alignment with the efforts on miniaturizing the components of electronic devices with enhanced performance, we investigate a dielectric nanocapacitor (DNC) based on metallic borophene electrodes separated with insulating hexagonal boron nitride (h-BN) monolayers $(n=1-5)$. The capacitive performance of the proposed DNC as a function of applied electric field $(\vec{E})$ and thickness of the dielectric material is examined by using $a b$ initio methods. The borophene plates and h-BN monolayers are commensurate and coupled only with van der Waals interaction, which constitutes an ideal configuration as a DNC. It is found that a single h-BN layer is not thick enough as a spacer to hinder quantum tunneling effects, and similar to the case with no insulating layer, borophene electrodes are shorted. Being effective from two h-BN layers, the charge separation on borophene plates is attained via $\vec{E}$ in the vertical direction. The capacitance of the DNC rapidly saturates at $\vec{E} \geqslant 0.1 \mathrm{~V} / \AA$ and reaches its maximum value of $0.77 \mu \mathrm{F} / \mathrm{cm}^{2}$ for $n=2$. The capacitance decreases with an increasing number of insulating layers as the distance between electrodes enlarges and shows a similar trend that is expected from the classical Helmholtz model. Our results suggest metallic and lightweight borophene and insulating h-BN monolayers as ideal constituents for the DNC design.
\end{abstract}

DOI: 10.1103/PhysRevMaterials.5.124002

\section{INTRODUCTION}

Recent advancements in nanoscience and engineering have miniaturized the elements of electronic devices with desired functionalities. In this respect, nanoscale capacitors have been realized and proposed as new generation charge and energy storage platforms [1-5]. In addition to having high power densities, nanocapacitors are more durable, have shorter charging times, and release stored charge faster than traditional capacitors and batteries [6-8]. These superior features suggest nanocapacitors as optimal components for diverse applications [9], and an increasing number of studies has been intensified in this field [3-5,8,10,11]. Different from electrochemical double-layer capacitors (EDLC), recently, dielectric nanocapacitors (DNCs) have been proposed, and remarkable progress has been achieved [12]. In the DNCs, instead of a liquid electrolyte, a solid dielectric is placed between the metallic plates (electrodes), providing the system more compactness and flexibility [13-15].

Since the size and performances of nanocapacitors are limited by their active components, in addition to other lowdimensional systems, two-dimensional (2D) materials have also been considered as conductive plates. The high surface area and good electrical conductivity of metallic 2D systems make them promising electrodes for both EDLC and DNCs. In this respect, graphene-based DNCs have already been proposed $[12,16,17]$. In this design, a dielectric material is used

\footnotetext{
*durgun@unam.bilkent.edu.tr
}

between the graphene electrodes, and the capacitance can be controlled with the thickness of the dielectric material (i.e., number of layers) without increasing the dimensions of the structure. On the other hand, unlike a traditional metal electrode, the quantum capacitance of graphene is limited by its low electronic density of states near the Fermi level [18-20]. This has led researchers to alternative capacitance materials that are directly taking into account the internal electronic structure of the electrodes. For instance, silicene has been suggested as an electrode material, and Yang et al. [16] have explored the effect of vacancies and dopants on quantum capacitance. They have shown that the quantum capacitance of silicene is larger than that of graphene, and it can be further increased by impurities [16].

In the quest of alternative 2D electrodes, borophene, which has been realized recently [21,22], can be a proper candidate [23]. It is stronger and more flexible than graphene, and has good electronic conductivity and superior ion transport properties [24-26]. Although 2D boron sheets have been proposed as an ideal anode material for alkali-ion batteries, borophenebased nanocapacitors have not been studied yet. In addition, hexagonal boron nitride (h-BN) multilayers, which are lattice matched with borophene, are wide band gap insulators and can serve as a proper dielectric medium between electrodes. With this motivation, in this paper, we design and characterize a DNC based on borophene electrodes, which are separated with insulating h-BN monolayers $(n=1-5)$. First, a commensurate heterostructure is formed, and optimized structures of the DNC are obtained up to $n=5$. Next, the variation of layer-dependent energy and excess charge is investigated 
as a function of an applied electric field $(\vec{E})$. Finally, the corresponding capacitance values (per area and per mass) are calculated considering two different formulations for each case, and obtained values are compared with the classical model.

\section{METHODOLOGY}

We performed first-principles calculations using the Vienna $a b$ initio simulation package [27-30] based on density functional theory (DFT). A plane-wave basis set with a kinetic energy cutoff of $550 \mathrm{eV}$ was utilized, and the exchangecorrelation potential was approximated by generalized gradient approximation with the Perdew-Burke-Ernzerhof functional [31]. Atomic positions and lattice constants were optimized by applying the conjugate gradient method. The Brillouin zone integration was performed with a $12 \times 16 \times$ $1 k$-point mesh using the Monkhorst-Pack method [32]. A vacuum spacing of $20 \AA$ was set along the perpendicular direction to the capacitor plane to hinder spurious interactions (see Fig. S1 within the Supplemental Material [33] for the schematic of supercell thickness, vacuum space, and distance between electrodes). In the geometrical optimization, the maximum Hellmann-Feynman force acting on each atom was less than $0.005 \mathrm{eV} / \AA$, and the energy convergence criteria between the two consecutive steps were taken to be $10^{-7} \mathrm{eV}$. The DFT-D2 method [34] was used for the van der Waals (vdW) interactions between weakly bound 2D layers. The electric field $(\vec{E})$ was applied by adding an artificial dipole sheet in the middle of the vacuum in the unit cell [35]. Due to the plane-wave basis description, this artificial dipole sheet can overlap with the charge of the layers if appropriate vacuum thickness that avoids the field emission into the vacuum is not taken into account [36]. The convergence of vacuum thickness was controlled by the differential charge density profile as presented in Fig. S3 within the Supplemental Material [33]. In addition, the data computed at varying vacuum spacing is reported in Table S1 within the Supplemental Material [33]. The charge separation on the electrodes was investigated by using the Bader charge analysis [37-39] and the density derived electrostatic and chemical approach [40] (Fig. S4 within the Supplemental Material [33]).

The energy difference between the total energies of the system [41] in the presence and absence of $\vec{E}$ is given by

$$
E_{C}(\vec{E})=E_{T}(\vec{E})-E_{T}(\vec{E}=0) .
$$

$E_{T}(\vec{E})$ and $E_{T}(\vec{E}=0)$ are calculated with same parameters and refer to the same energy reference (and normalized with respect to the DNC supercell). As the dimensions of the DNC are within the nanometer scale, the total energies should be calculated by $a b$ initio methods [41]. This energy difference corresponds to stored energy $\left(E_{C}\right)$ in the DNC only within the capacitive regime.

The gravimetric capacitance of the system can be calculated as

$$
C_{E}=\frac{\left(\Delta Q^{2}\right)}{2 m E_{C}},
$$

where $\Delta Q$ is the the excess charge on borophene layers (metal electrodes) due to the applied electric field $[\Delta Q=Q(E \neq$
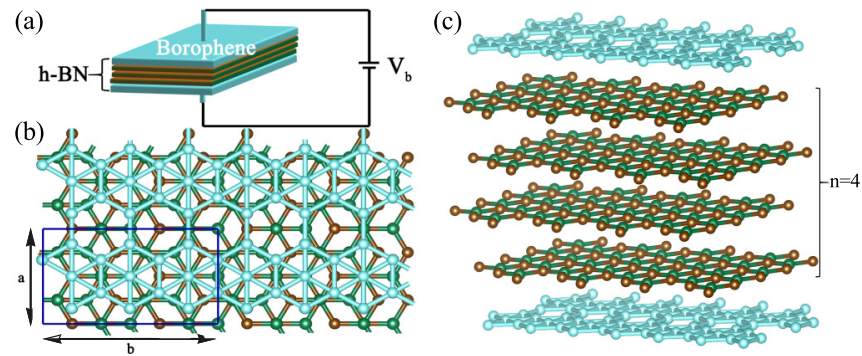

FIG. 1. (a) The schematic of the DNC based on borophene and h-BN monolayers, (b) top and (c) side views of the capacitor model for four h-BN monolayers. The borophene and h-BN layers are represented with light blue and green-brown colors.

$0)-Q(E=0)]$ and $m$ is the total mass of the system including $\mathrm{B}$ and $\mathrm{N}$ atoms. The capacitance value can also be estimated as

$$
C_{\Delta V}=\frac{\Delta Q}{m \Delta V_{z}},
$$

where $V_{z}$ is the plane-averaged potential in the vertical direction and $\Delta V_{z}$ is the potential difference between electrodes in the presence of $\vec{E}$ (Figs. S5 and S7 within the Supplemental Material [33]). In addition, the classical Helmholtz expression $C_{H}=\kappa \varepsilon_{0}(A / m d)$ (where $\kappa$ is the calculated dielectric constant of the h-BN layer(s) [42], $\varepsilon_{0}$ is the permittivity of free space, $A$ is the area of the borophene layer in the unit cell, and $d$ is the distance between electrodes) is considered for comparison.

To further test the reliability of the methodology, the capacitance of the system was also calculated with the OPENMX code [43] based on pseudoatomic localized basis functions, and the obtained results are shown in Fig. S8 within the Supplemental Material [33].

\section{RESULTS AND DISCUSSION}

To form the DNCs, we consider planar and metallic polymorphs of borophene $\left(\beta_{12}\right)[22,44]$ as electrodes and insulating h-BN monolayers $(n=1-5)$ as the dielectric medium [45]. The schematic of the designed DNC, which has the lowest energy stacking configuration, is shown in Fig. 1, and the supercell is illustrated in Fig. S1 within the Supplemental Material [33]. In this model, $3 \times 1$ borophene and $2 \times 2 \mathrm{~h}$-BN rectangular supercells are taken to ensure the lattice match. The calculated lattice constants are $\mathbf{a}=8.78$ and $\mathbf{b}=5.07 \AA$ for free-standing borophene, $\mathbf{a}=8.70$ and $\mathbf{b}=5.02 \AA$ for h-BN. Accordingly, these layers are commensurate and form a stable heterostructure (Fig. S2 within the Supplemental Material [33]) having $\mathbf{a}=8.72$ and $\mathbf{b}=5.03 \AA$. The DNC is relaxed for each configuration (i.e., varying h-BN monolayers), but the change in the lattice constants is found to be negligible. It should be noted that the layers of the DNC are coupled by vdW interaction and no vertical bond formation is noted even when various initial configurations are tested.

Next, the electronic band structure of the DNC in the absence and presence of $\vec{E}$ along the $z$ direction is calculated. The obtained results for the borophene bilayer (without the 

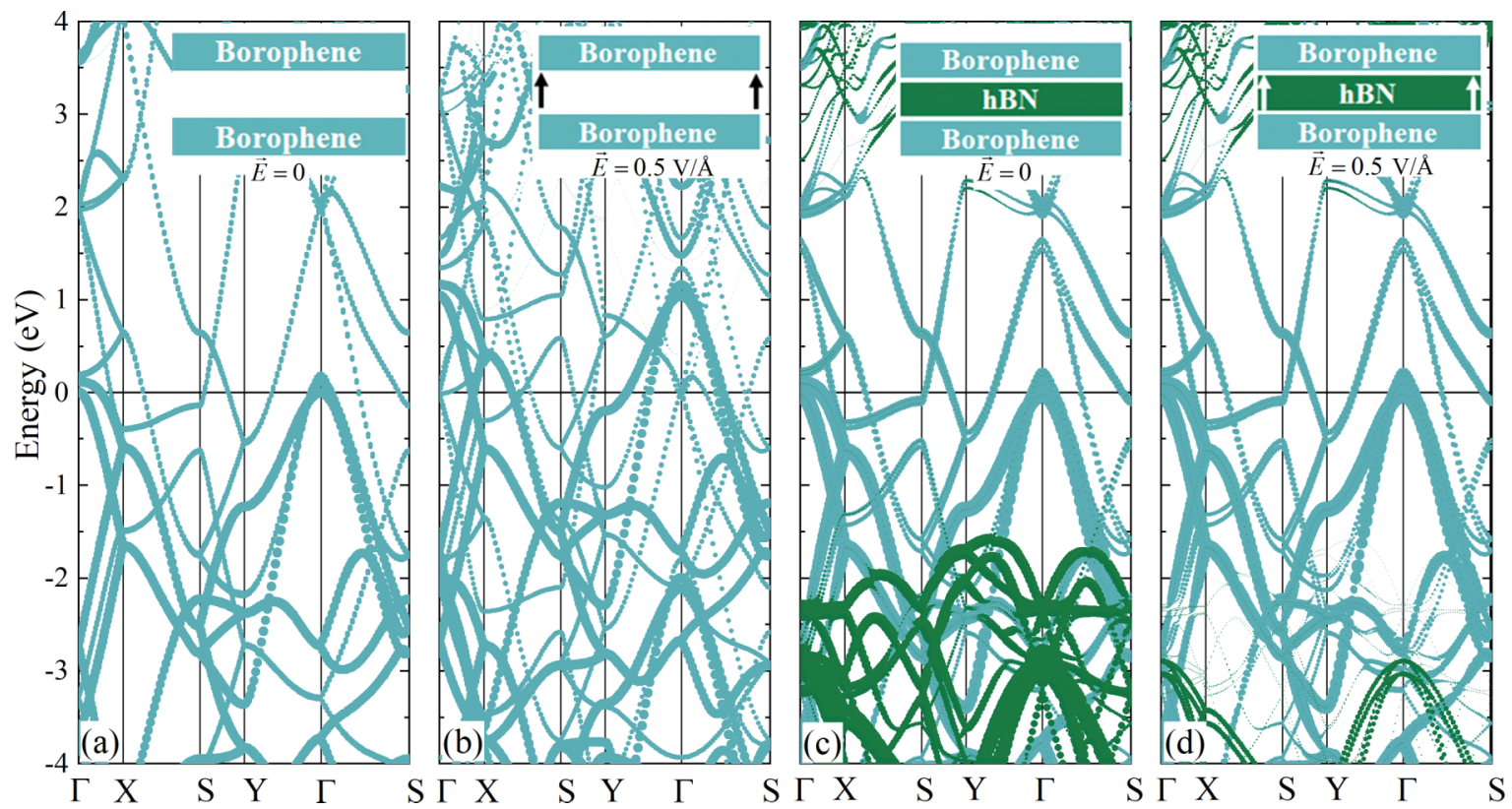

FIG. 2. The projected band structures of the borophene bilayer without an insulating spacer (a) for $\vec{E}=0$ and (b) $\vec{E}=0.5 \mathrm{~V} / \AA$, and the projected band structures of the DNC with two h-BN layers (c) for $\vec{E}=0$ and (d) $\vec{E}=0.5 \mathrm{~V} / \AA$.

h-BN monolayer) and for the DNC with two h-BN monolayers at $\vec{E}=0$ and $0.5 \mathrm{~V} / \AA$ are shown in Fig. 2. The projected band structures for $n=2$ are presented as an illustration, and similar results are obtained when $n$ increases. When $\vec{E}$ is applied, a built-in potential $\left(\Delta V_{z}\right)$ accommodates excess electric charge on the top borophene layer and depletes the same amount of charge from the bottom layer, preserving the overall charge neutrality. Borophene bilayers retain their metallicity even when the band structure is modified upon applied $\vec{E}$ [Figs. 2(a) and 2(b)]. For the DNC with $n=2$, due to the weak coupling between the layers, the characteristics of borophene and h-BN layers can be recognized separately. It is clear from Fig. 2(c) that metallic borophene layers have contributions to the electronic bands around the Fermi level and a large band gap is noted for the h-BN bilayer. In the presence of $\vec{E}$, the DNC is still metallic, and dielectric layers remain insulating. If $\vec{E}$ is further increased (i.e., up to the breakdown voltage), resonant tunneling between borophene electrodes over the dielectric medium can be realized [46]. In addition, increasing $\vec{E}$ can yield electron field emission into the vacuum $[36,47]$ due to the computational implementation of the electric field [35]. The field emission mechanism can be followed from the differential charge density (Fig. S3 within the Supplemental Material [33]) along the $z$ direction, which should not have substantial charge accumulation around the vacuum region when the electric field is switched on. Accordingly, the electric field range is restricted to $\vec{E} \leqslant 0.6 \mathrm{~V} / \AA$ at which the spurious charge escapes into vacuum is very small and does not influence the obtained results.

To investigate the capacitive response of the DNC, the variation of the stored energy $\left(E_{C}\right)$ and excess charge $(\Delta Q)$ per DNC supercell as a function of $\vec{E}$ are calculated for $n=2-5$ (Fig. 3). Interestingly, the total energy difference $\left(E_{\text {diff }}\right)$ in the presence and absence of an electric field for $n=1$ exhibits the same behavior as the borophene bilayer without a spacer $(n=0)$, and it increases rapidly with elevated $\vec{E}$ as shown in the inset of Fig. 3(a). This response can be explained by the quantum tunneling phenomena where the electric field causes dielectric breakdown depending on the thickness of dielectric material such that this regime vanishes with the increasing number of the h-BN layer. Accordingly, as there is no capacitive response, it is not meaningful to define stored energy within this regime. Similar phenomena were also observed at the layered van der Waals $2 \mathrm{D}$ heterostructures, i.e., h-BN/graphene/h-BN [48] and ZnSe/AlAs [49]. In order to clarify this effect, the application of an external electric field can be defined via the bias potential (i.e., $E_{z}=V_{b} / d$, where $d$ is the distance between borophene plates as shown in Fig. 1). By the influence of $V_{b}$, the charge separation between plates yields a built-in potential $\left(\Delta V_{z}\right)$ due to the potential drop between borophene layers (the electric field within the DNC can be also obtained by considering $\Delta V_{z}$ ). The variation of $\Delta V_{z}$ is exactly the same as $V_{b}$ for the system without the spacer and a single h-BN layer. In other words, the applied bias potential induces approximately the same amount of built-in potential, and this confirms the tunneling effect (Fig S6 within the Supplemental Material [33]). As expected, with the increasing number of h-BN layers, the alternation of $\Delta V_{z}$ becomes incomparable with $V_{b}$ (and the electric field within the DNC decreases substantially) indicating the effect of the dielectric medium. In that sense, meaningful capacitive properties of the DNC can be estimated for $n \geqslant 2$. As shown in Fig. 3(a), $E_{c}$ increases with the magnitude of the electric field and with the number of layers for $n=2-5$.

Figure 3(b) presents the variation of the magnitude of the stored charge $(\Delta Q)$ in borophene layers as a function of $\vec{E}$. It is noted that $\Delta Q$ linearly increases with $\vec{E}$ and almost independent of the number of h-BN monolayers for $n=2-5$. The dielectric medium allows charge separation on the borophene plates (same amount with an opposite sign) by $\vec{E}$ compatible with the nature of dielectric capacitors. For the borophene bilayer, $\Delta Q$ is almost zero in the presence of $\vec{E}$, and for 

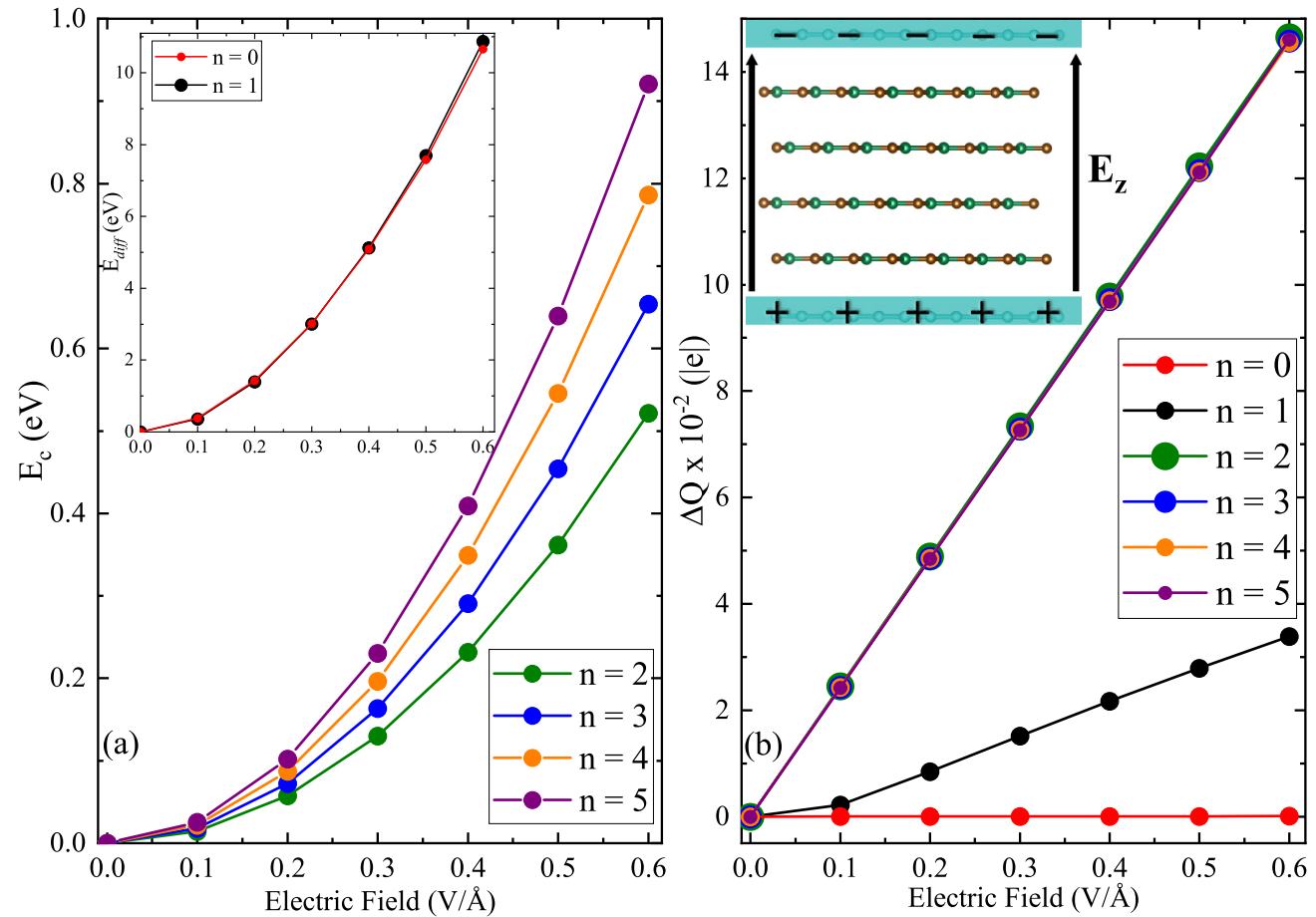

FIG. 3. (a) Variation of the stored energy $E_{c}$ and (b) excess charge $(\Delta Q)$ as a function of the external electric field for $n=2-5$. Variation of total energy difference $\left(E_{\text {diff }}\right)$ in the presence and absence of an applied field for $n=0$ and 1 is shown as an inset.

$n=1$, it slowly increases with $\vec{E}$ since the system is shorted as discussed above.

Finally, the capacitance per area $\left(C_{E}\right)$ is calculated according to Eq. (2) as a function of $\vec{E}$ for varying $n$ as shown in Fig. 4(a). $C_{E}$ rapidly saturates for $\vec{E} \geqslant 0.1 \mathrm{~V} / \AA$ for all cases and expectedly become independent of the ap- plied field. The maximum value of $C_{E}$ is obtained for $n=$ 2 , and it monotonically decreases with increasing $n$ as the distance between borophene plates enlarges (converging to the $1 / d$ trend as shown in Fig. S8 within the Supplemental Material [33]). $C_{E}$ takes values between $0.77-0.42 \mu \mathrm{F} / \mathrm{cm}^{2}$ and $(0.84-2.75 \mathrm{~F} / \mathrm{g}$ ) for $n=2-5$, which is comparable with
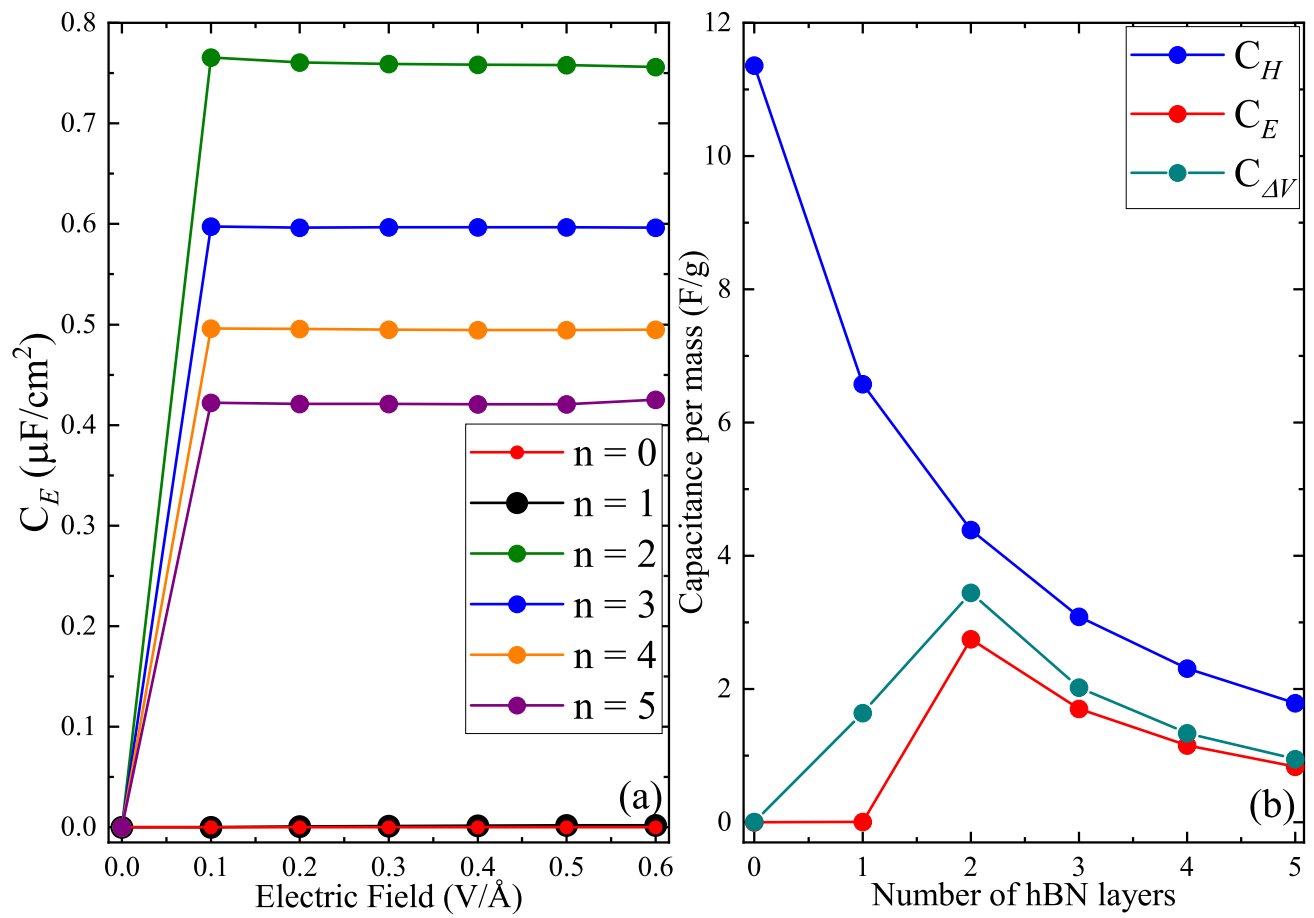

FIG. 4. (a) The capacitance $\left(\mathrm{C}_{E}\right)$ as a function of applied electric field for $n=1-5$, (b) the variation of capacitance calculated with two different definitions $\left(C_{E}\right.$ and $\left.C_{\Delta V}\right)$ and the classical Helmholtz model $\left(C_{H}\right)$ with the number of h-BN layers. 
TABLE I. Number of h-BN layers between the borophene layer $n$; distance between borophene layers $d$; total mass of the unit cell $m$; dielectric constants of BN layers (taken from Ref. [42]), $\kappa$; excess charge on plates $\Delta Q$; stored energy $E_{c}$; plane averaged potential difference between borophene layers $\Delta V_{z}$; capacitance $\left(C_{E}\right)$ per area $\left(A=43.5 \times 10^{-20} \mathrm{~m}^{2}\right)$ and mass $(m)$; capacitance $\left(C_{\Delta V}\right)$ per mass $(m)$. $\Delta Q, E_{c}$, and $\Delta V_{z}$ values are evaluated at $\vec{E}=0.5 \mathrm{~V} / \AA$.

\begin{tabular}{cccccccccc}
\hline \hline$n$ & $d(\AA)$ & $m\left(\mathrm{~kg} \times 10^{-22}\right)$ & $\kappa$ & $\Delta Q\left(|e| \times 10^{-2}\right)$ & $E_{c}(\mathrm{eV})$ & $\Delta V_{z}(\mathrm{eV})$ & $C_{E}\left(\mu \mathrm{F} / \mathrm{cm}^{2}\right)$ & $C_{E}(\mathrm{~F} / \mathrm{g})$ & $C_{\Delta V}(\mathrm{~F} / \mathrm{g})$ \\
\hline 0 & 6.29 & 5.39 & 1.00 & 0.004 & 7.590 & 3.15 & $3.87 \times 10^{-9}$ & $1.13 \times 10^{-7}$ & $7.08 \times 10^{-3}$ \\
1 & 6.33 & 8.68 & 3.29 & 2.79 & 7.700 & 3.15 & $1.86 \times 10^{-3}$ & $9.71 \times 10^{-3}$ & 1.64 \\
2 & 9.43 & 11.98 & 3.44 & 12.23 & 0.362 & $0.62 \times 10^{-2}$ & 0.76 & 2.75 \\
3 & 12.48 & 15.28 & 3.52 & 12.15 & 0.454 & $1.30 \times 10^{-2}$ & 0.60 & 1.71 \\
4 & 15.54 & 18.65 & 3.58 & 12.13 & 0.546 & $2.04 \times 10^{-2}$ & 0.49 & 1.16 \\
5 & 18.70 & 21.94 & 3.62 & 12.11 & 0.639 & $3.80 \times 10^{-2}$ & 0.42 & 0.84 \\
\hline \hline
\end{tabular}

the reported capacitance values for graphene-based electrodes $\left(0.2-0.3 \mu \mathrm{F} / \mathrm{cm}^{2}\right)[20]$ but lower than that of $\mathrm{Au} / \mathrm{h}-\mathrm{BN} / \mathrm{Au}$ $\left(0.14-8.74 \mu \mathrm{F} / \mathrm{cm}^{2}\right)[50]$, graphene $/ \mathrm{h}-\mathrm{BN} /$ graphene $(5.6-$ $24.5 \mathrm{~F} / \mathrm{g})$ [12], graphyne/h-BN/graphyne $(20-40 \mathrm{~F} / \mathrm{g})[51]$ heterostructures, and graphene-based supercapacitors (100$250 \mathrm{~F} / \mathrm{g}$ ) [52]. The obtained values for pristine borophene can be further increased with functionalization [53], doping [20,54-58], and defects or impurities [59]. For $n<2, C_{E} \sim 0$ as the borophene plates are shorted. In addition, the gravimetric capacitance is also calculated by considering Eq. (3) $\left(C_{\Delta V}\right)$ and by the classical Helmholtz expression $\left(C_{H}\right)$ by the relevant parameters given in Table I. (The dielectric constant of h-BN monolayers is taken from Ref. [42] and can be estimated by using the classical electrostatic energy definition). The obtained results are qualitatively comparable and show a similar trend with respect to the number of h-BN layers for $n \geqslant 2$ as shown in Fig. 4(b). It is noted that the profile of the DNC converges to a classical capacitor beyond the quantum tunneling regime as the distance between the electrodes increases. As expected, $C_{H}$ decreases monotonically with $n$ since the quantum effect for $n=1$ (and for the borophene bilayer without a spacer, i.e., $n=0$ ) cannot be revealed by a classical approach.

Additionally, the capacitance of the designed heterosystem is also calculated by using the localized basis set and the results are shown in Fig. S8 within the Supplemental Material [33]. These results indicate that the magnitude of the capacitance slightly increases (due to the better description of excess charge) with respect to the plane-wave approach, however, they follow the same trend. Moreover, the data obtained with both of the methodologies converge to capacitance values estimated with the Helmholtz formula as $d$ increases.

\section{CONCLUSION}

To summarize, we designed a DNC based on planar borophene electrodes and insulating h-BN monolayers and calculate the capacitive properties of the system with respect to the thickness of the dielectric medium (i.e., the number of h-BN layers $n$ ). It is found that borophene and h-BN plates are stable, commensurate, and interact via van der Waals forces, which form an ideal configuration as a DNC. The applied electric field along the vertical direction results in charge separations on borophene electrodes in compliance with the character of dielectric capacitors. It is found that the single h-BN layer is not thick enough as an insulating medium to prevent quantum tunneling effects and similar to the borophene bilayer without a spacer, borophene electrodes are shorted. For $n \geqslant 2$, stored energy $\left(E_{C}\right)$ and excess charge on plates $(\Delta Q)$ increases with applied $\vec{E}$. Although $\Delta Q$ is nearly independent of the number of h-BN monolayers, $E_{C}$ increases with $n$. The capacitance of the DNC rapidly saturates for $\vec{E} \geqslant$ $0.1 \mathrm{~V} / \AA$ and reaches its maximum value of $0.77 \mu \mathrm{F} / \mathrm{cm}^{2}$ $(2.75 \mathrm{~F} / \mathrm{g})$ for $n=2$, which is comparable with the capacitance value of graphene-based nanocapacitors. Capacitance decreases with increasing insulating layers as the distance between electrodes enlarges and converges to a similar trend that is expected from a classical Helmholtz model. The obtained data with the plane-wave approach is further confirmed with using a localized-basis set, and the same trends are revealed. Our results indicate that metallic borophene and insulating h-BN are ideal components of nanocapacitors, and the proposed DNC model can be utilized in nanoelectronic applications.

\section{ACKNOWLEDGMENTS}

This work was supported by the Scientific and Technological Research Council of Turkey (TUBITAK) under Project No. 117F383. The calculations were performed at TUBITAK ULAKBIM, High Performance and Grid Computing Center (TR-Grid e-Infrastructure) and the National Center for High Performance Computing of Turkey (UHeM) under Grant No. 5007092019. Y.M. and A.M. acknowledge the Ankara University for the High Performance Computing Facility through the AYP under Grant No. 17A0443001.
[1] K. Uprety, L. Ocola, and O. Auciello, J. Appl. Phys. 102, 084107 (2007).

[2] C. Liu, F. Li, L.-P. Ma, and H.-M. Cheng, Adv. Mater. 22, E28 (2010).
[3] L. C. Haspert, S. B. Lee, and G. W. Rubloff, ACS Nano 6, 3528 (2012).

[4] L. Wei, Q.-X. Liu, B. Zhu, W.-J. Liu, S.-J. Ding, H.-L. Lu, A. Jiang, and D. W. Zhang, Nanoscale Res. Lett. 11, 213 (2016). 
[5] M. Stengel and N. A. Spaldin, Nature (London) 443, 679 (2006).

[6] K. H. An, W. S. Kim, Y. S. Park, J.-M. Moon, D. J. Bae, S. C. Lim, Y. S. Lee, and Y. H. Lee, Adv. Funct. Mater. 11, 387 (2001).

[7] J. Carlberg and O. Inganäs, Electrochem. Soc. 144, L61 (1997).

[8] S. Ekanayake, M. Ford, and M. Cortie, Materials Forum 27, 15 (2004).

[9] S. Saha, M. DaSilva, Q. Hang, T. Sands, and D. Janes, Nanotechnology 17, 2284 (2006).

[10] N. Engheta, A. Salandrino, and A. Alu, Phys. Rev. Lett. 95, 095504 (2005).

[11] J. I. Sohn, Y.-S. Kim, C. Nam, B. Cho, T.-Y. Seong, and S. Lee, Appl. Phys. Lett. 87, 123115 (2005).

[12] V. O. Özçelik and S. Ciraci, J. Phys. Chem. C 117, 15327 (2013).

[13] G. Yue, X. Ma, W. Zhang et al., Nanoscale Res. Lett. 10, 1 (2015).

[14] I. Shown, A. Ganguly, L.-C. Chen, and K.-H. Chen, Energy Sci. Eng. 3, 2 (2015).

[15] A. Yu, I. Roes, A. Davies, and Z. Chen, Appl. Phys. Lett. 96, 253105 (2010).

[16] G. Yang, Q. Xu, X. Fan, and W. Zheng, J. Phys. Chem. C 122, 1903 (2018).

[17] J. Xia, F. Chen, J. Li, and N. Tao, Nat. Nanotechnol. 4, 505 (2009).

[18] C. Zhan, J. Neal, J. Wu, and D.-E. Jiang, J. Phys. Chem. C 119, 22297 (2015).

[19] P. A. Brooksby, A. K. Farquhar, H. M. Dykstra, M. R. Waterland, and A. J. Downard, J. Phys. Chem. C 119, 25778 (2015).

[20] J. Vatamanu, X. Ni, F. Liu, and D. Bedrov, Nanotechnology 26, 464001 (2015).

[21] A. J. Mannix, X.-F. Zhou, B. Kiraly, J. D. Wood, D. Alducin, B. D. Myers, X. Liu, B. L. Fisher, U. Santiago, J. R. Guest et al., Science 350, 1513 (2015).

[22] B. Feng, J. Zhang, Q. Zhong, W. Li, S. Li, H. Li, P. Cheng, S. Meng, L. Chen, and K. Wu, Nat. Chem. 8, 563 (2016).

[23] C. Zhan, P. Zhang, S. Dai, and D.-E. Jiang, ACS Energy Lett. 1, 1241 (2016).

[24] H. Banda, S. Périé, B. Daffos, P.-L. Taberna, L. Dubois, O. Crosnier, P. Simon, D. Lee, G. De Paëpe, and F. Duclairoir, ACS Nano 13, 1443 (2019).

[25] Y. Korenblit, A. Kajdos, W. C. West, M. C. Smart, E. J. Brandon, A. Kvit, J. Jagiello, and G. Yushin, Adv. Funct. Mater. 22, 1655 (2012).

[26] B. G. Choi, J. Hong, W. H. Hong, P. T. Hammond, and H. Park, ACS Nano 5, 7205 (2011).

[27] G. Kresse and J. Hafner, Phys. Rev. B 47, 558 (1993).

[28] G. Kresse and J. Hafner, Phys. Rev. B 49, 14251 (1994).

[29] G. Kresse and J. Furthmüller, Comput. Mater. Sci. 6, 15 (1996).

[30] G. Kresse and J. Furthmüller, Phys. Rev. B 54, 11169 (1996).
[31] J. P. Perdew, K. Burke, and M. Ernzerhof, Phys. Rev. Lett. 77, 3865 (1996).

[32] H. J. Monkhorst and J. D. Pack, Phys. Rev. B 13, 5188 (1976).

[33] See Supplemental Material at http://link.aps.org/supplemental/ 10.1103/PhysRevMaterials.5.124002 for additional details on the schematic of the supercell, structural stability of the heterostructure, differential charge density analysis, comparison of charge analysis methods, plane average potential $\left(V_{z}\right)$, bias potential $\left(V_{b}\right)$, and OpenMX results.

[34] S. Grimme, J. Comp. Chem. 27, 1787 (2006).

[35] J. Neugebauer and M. Scheffler, Phys. Rev. B 46, 16067 (1992).

[36] P. J. Feibelman, Phys. Rev. B 64, 125403 (2001).

[37] W. Tang, E. Sanville, and G. Henkelman, J. Phys. Condens. Matter 21, 084204 (2009).

[38] E. Sanville, S. D. Kenny, R. Smith, and G. Henkelman, J. Comput. Chem. 28, 899 (2007).

[39] G. Henkelman, A. Arnaldsson, and H. Jónsson, Comput. Mater. Sci. 36, 354 (2006).

[40] N. G. Limas and T. A. Manz, RSC Adv. 6, 45727 (2016).

[41] The total energy accounts for the total energy of the supercell including vacuum and not just for the DNC region.

[42] A. Laturia, M. L. Van de Put, and W. G. Vandenberghe, npj 2D Mater. Appl. 2, 6 (2018).

[43] T. Ozaki, K. Nishio, and H. Kino, Phys. Rev. B 81, 035116 (2010).

[44] P. Ranjan, T. K. Sahu, R. Bhushan, S. S. Yamijala, D. J. Late, P. Kumar, and A. Vinu, Adv. Mater. 31, 1900353 (2019).

[45] K. K. Kim, A. Hsu, X. Jia, S. M. Kim, Y. Shi, M. Dresselhaus, T. Palacios, and J. Kong, ACS Nano 6, 8583 (2012).

[46] C. Zener, Proc. R. Soc. London A 145, 523 (1934).

[47] Y. Mogulkoc, M. Modarresi, A. Mogulkoc, and B. Alkan, Phys. Rev. Appl. 12, 054036 (2019).

[48] A. Valsaraj, L. F. Register, E. Tutuc, and S. K. Banerjee, J. Appl. Phys. 120, 134310 (2016).

[49] F. Yao, X. Zhou, and A. Xiong, Appl. Phys. A 126, 1 (2020).

[50] G. Shi, Y. Hanlumyuang, Z. Liu, Y. Gong, W. Gao, B. Li, J. Kono, J. Lou, R. Vajtai, P. Sharma et al., Nano Lett. 14, 1739 (2014).

[51] B. Bhattacharya, U. Sarkar, and N. Seriani, J. Phys. Chem. C 120, 26579 (2016).

[52] C. Liu, Z. Yu, D. Neff, A. Zhamu, and B. Z. Jang, Nano Lett. 10, 4863 (2010).

[53] S. M. Mousavi-Khoshdel and E. Targholi, Carbon 89, 148 (2015).

[54] M. Mousavi-Khoshdel, E. Targholi, and M. J. Momeni, J. Phys. Chem. C 119, 26290 (2015).

[55] S.-S. Yu and W.-T. Zheng, Nanoscale 2, 1069 (2010).

[56] E. Paek, A. J. Pak, K. E. Kweon, and G. S. Hwang, J. Phys. Chem. C 117, 5610 (2013).

[57] C. Wang, Y. Zhou, L. Sun, Q. Zhao, X. Zhang, P. Wan, and J. Qiu, J. Phys. Chem. C 117, 14912 (2013).

[58] D. Jana, C.-L. Sun, L.-C. Chen, and K.-H. Chen, Prog. Mater. Sci. 58, 565 (2013).

[59] A. J. Pak, E. Paek, and G. S. Hwang, Carbon 68, 734 (2014). 